\title{
El canal de potasio dependiente de voltaje Kv10.1 y el cáncer
}

\author{
Walter Stühmer \\ Instituto Max-Planck para Medicina Experimental, Göttingen, Alemania \\ Artículo de posesión para el ingreso como miembro correspondiente de la \\ Academia Colombiana de Ciencias Exactas, Físicas y Naturales el 21 de septiembre de 2017
}

\begin{abstract}
Resumen
Entre los más de 100 genes conocidos que codifican canales iónicos selectivos de potasio, el llamado Kv10.1 posee propiedades muy particulares que lo diferencian de los demás. El canal Kv10.1 es dependiente del voltaje y prácticamente se detecta únicamente en tejido nervioso. Sorprendentemente, se descubrió que se encuentra en más del $70 \%$ de los cánceres humanos, y en tejidos de rápido crecimiento como la placenta, las células germinales del testículo o las criptas del colon. Su distribución subcelular ayudó a revelar su función en el ciclo celular, a lo largo del cual es regulado por factores de crecimiento y por los genes supresores de tumores $p 53$ y RB1. El Kv10.1 también favorece la internalización del cilio primario, paso indispensable para la división celular. Dado que las células cancerosas se dividen rápidamente o presentan alteraciones en la función de los factores de crecimiento o en los mencionados genes, suelen expresar el Kv10.1, lo cual se puede detectar usando anticuerpos que actúan contra él. Los pacientes en cuyos tumores se detectan altos niveles de Kv10.1 tienen un peor pronóstico que aquellos con niveles bajos. Además, el bloqueo de la función del Kv10.1 permite reducir la proliferación celular, lo cual lo convierte en un nuevo marcador diagnóstico del cáncer, y en un blanco para su tratamiento. (C) 2017. Acad. Colomb. Cienc. Ex. Fis. Nat.
\end{abstract}

Palabras clave: Canales iónicos; Cambio conformacional dependiente de voltaje; División celular; Cáncer; Cilio primario.

The voltage-dependent potassium channel Kv10.1 and cancer

\begin{abstract}
Among the over 100 genes that encode for the various potassium channels known so far, the Kv10.1 exhibits properties that are quite unique. It is voltage-dependent and expressed almost exclusively in the nervous system. Surprisingly, it was found overexpressed in more than $70 \%$ of human cancer tissue of diverse origin, as well as in fast growing tissue such as placenta, germinal cells of testicles and in colon crypts. Its sub-cellular distribution allowed for elucidating its role in the cell cycle, during which it is regulated by growth factors and tumor suppressor genes such as the $p 53$ and the RB1. In addition, Kv10.1 favors the internalization of the primary cilium, which is essential for cell division. Given that tumor cells grow and divide rapidly because they often have defective growth-factor signaling or defects in one of the mentioned genes, they frequently over-express Kv10.1, which can be detected using specific antibodies. Patients with high levels of Kv10.1 in their tumors have worse prognosis than those with low levels. In addition, blocking the function of Kv10.1 allows reducing cell proliferation. Therefore, Kv10.1 offers a novel diagnostic and therapeutic window in cancer treatment. (C) 2017. Acad. Colomb. Cienc. Ex. Fis. Nat.
\end{abstract}

Key words: Ion channels; Voltage-gating; Cell division; Cancer; Primary cilium.

\section{Introducción}

Los canales iónicos son proteínas de la transmembrana que permiten el intercambio de iones y ejercen funciones de relevancia fisiológica, como la homeostasis iónica, el establecimiento del potencial de reposo y la modulación de la excitabilidad en tejidos excitables, en especial los neuronales y musculares. Dichos canales se dividen en dos clases principalmente: los dependientes de voltaje, como los rectificadores tardíos, los rectificadores anómalos o los transitorios, y los activados por ligandos, como los activados por el calcio o el trifosfato de adenosina (ATP). Mediante técnicas de ADN recombinante en mutaciones del gen shaker de Drosophila melanogaster, tres grupos de investigación (Schwarz, et al.,1988, Pongs, et al., 1988, Kamb, et al., 1988) lograron elucidar la secuencia de aminoácidos de una familia de canales de potasio dependientes de voltaje presentes en el cerebro de ratones (Stuhmer, et al., 1988). Actualmente se conocen unas 80 variedades de estos canales pertenecientes a la familia shaker. Partiendo de otra mutación de D. melanogaster llamada Ether a-go-go (debido a sus

\section{Correspondencia:}

Walter Stühmer,ws@em.mpg.de

Recibido: 25 de julio de 2017

Aceptado: 30 de agosto de 2017 
movimientos rítmicos al ser anestesiada con éter), se logró identificar otro canal de potasio dependiente de voltaje con propiedades inusuales con el cual se formó una nueva familia llamada eag (de Ether-a-go-go) y que en la nomenclatura oficial se conoce como Kv10.1. La "K" indica que el canal es selectivo para potasio, la " $\mathrm{v}$ " que es dependiente de voltaje, y el número "10.1", que pertenece a la décima familia encontrada y es su primer miembro descrito. Además, se han encontrado dos homólogos relevantes, el Kv10.2 y otro canal cuya función fisiológica en el corazón ya se conocía, al cual se denominó Kv11.1 (Warmke \& Ganetzky, 1994).

Normalmente los canales de potasio se bloquean con el cesio, que al igual que el potasio pertenece a los metales alcalinos. Sorprendentemente, el Kv10.1 es permeable al cesio. Otra sorpresa fue encontrar que su velocidad de apertura (activación) depende en gran medida del potencial medio antes del pulso que causa la activación. Ello implica la existencia de una especie de "memoria" del canal, que "recuerda", por ejemplo, la actividad excitadora durante los $100 \mathrm{~ms}$ anteriores al estímulo. De hecho, se pudo demostrar que los ratones en los cuales se eliminó el gen que codifica el canal Kv10.1 son 'hiperexcitables'. Esta excitabilidad exagerada se debía a la ausencia de Kv10.1 en las terminales sinápticas del cerebelo localizadas entre las fibras trepadoras y las células de Purkinje (Mortensen, et al., 2015). Además, gracias a que el calcio intracelular modula el Kv10.1 (Stansfeld, et al., 1996), dicho canal regula la actividad motora dependiendo de la actividad previa; mucha actividad, lo cual conlleva la entrada de calcio, resulta en inhibición. Por lo tanto, la ausencia del Kv10.1 induce hiperactividad, pero no conlleva defectos del desarrollo ni alteraciones obvias del comportamiento en ratones genéticamente modificados para que no expresen el Kv10.1 (Ufartes, et al., 2013). La ausencia de alteraciones en el desarrollo probablemente se debe a que en los mamíferos el Kv10.1 comienza a expresarse después del nacimiento y solo se estabiliza cuando llegan a la edad adulta.

La gran dependencia de la cinética de activación del potencial anterior es una propiedad tan peculiar que permite determinar y aislar las corrientes del Kv10.1 de cualquier preparación que contenga muchos otros canales dependientes de voltaje. Esto ha sido de gran relevancia para los estudios posteriores, como veremos más adelante. Otra propiedad única del Kv10.1 es su modulación por el calcio intracelular, como ya se señaló (Stansfeld, et al., 1996) y el magnesio extracelular (Terlau, et al., 1996). Cabe recordar que el calcio es esencial para la progresión a través del ciclo celular, en especial para terminar las fases de síntesis de $\mathrm{ADN}(\mathrm{S})$ y mitótica $(\mathrm{M})$, y que las células cancerosas alteran su regulación por el calcio.

\section{¿Cuál es la posible función fisiológica del Kv10.1?}

Ya que la ausencia del Kv10.1 parece no causar trastornos graves ni durante la embriogénesis ni en el adulto, aparte de la modulación de la excitabilidad, ¿cuáles son sus otras funciones fisiológicas o fisiopatológicas?
Las funciones fisiológicas no se conocen con certeza. El comportamiento de la mutación Ether-a-go-go de $D$. melanogaster indica que, al menos en este insecto, la coordinación motora es regulada por el Kv10.1, aunque seguramente apenas hemos comenzado a descubrir sus verdaderas funciones. Recientemente, el papel de los canales iónicos, en general, (Arcangeli \& Becchetti, 2015), y específicamente de los canales de potasio (Pardo \& Stühmer, 2014) en el cáncer, han generado tal número de investigaciones que se ha originado un nuevo campo. El primer indicio de una función importante del Kv10.1 en el cáncer fue el descubrimiento de que tanto la permeabilidad como su nivel de expresión dependen del ciclo celular (Camacho, et al., 2000). Teniendo en cuenta la función del calcio en el ciclo celular y en el cáncer, y guiados por el descubrimiento de que el nivel expresión del Kv10.1 en las células tumorales está regulado por el gen supresor de tumores $p 53$, por el factor de crecimiento E2F1, el cual favorece la división celular, y por el micro-ARN miR-34a, se analizaron las regiones reguladoras del gen que codifica el Kv10.1. En la región promotora se pudieron encontrar elementos que responden al factor de crecimiento E2F1, y en la región no codificante 5 , , secuencias de unión para el miR-34a. Por lo tanto, la participación del Kv10.1 en el cáncer está directamente relacionada con uno de los factores más frecuentemente alterados en las células tumorales (p53). De hecho, usando técnicas genéticas para forzar la expresión del Kv10.1 en células que normalmente no lo expresan, no solo es posible estudiar las propiedades de estos canales, sino también sus efectos en los organismos. $\mathrm{Al}$ inyectar subcutáneamente células así transformadas en ratones inmunodeficientes (para que no rechacen las células incorporadas) se inducen tumores agresivos. En el estudio de más de 1.500 muestras de tejido de pacientes con cánceres de distinta índole, se pudo demostrar que en más del $70 \%$ de los casos se expresaba el Kv10.1 (Hemmerlein, et al., 2006, Menéndez, et al., 2012, Wadhwa, et al., 2009). La hipótesis de que el Kv10.1 está íntimamente relacionado con el cáncer ya está aceptada en la literatura. Además de ser un marcador precoz de células que se dividen rápidamente (Urrego, $\boldsymbol{e} t$ al., 2016), el Kv10.1 es un buen indicador de la agresividad del tumor y es útil para el pronóstico del paciente (Agarwal, et al., 2010, Lastraioli, et al., 2015, Martínez, et al., 2015). Una reciente revisión de gran parte de la temática aquí expuesta se encuentra en Ouadid-Ahidouch, et al., (2016).

\section{Mecanismos de regulación genética del Kv10.1}

En la figura 1 se ilustran los pasos individuales de la regulación marcados con letras que corresponden al texto en paréntesis. Las flechas verdes indican un efecto positivo, las rojas, uno negativo.

La expresión ectópica, es decir, fuera de lugar, del Kv10.1 favorece la progresión del ciclo celular, el cual es fundamental no solo para el desarrollo de tumores malignos y benignos, sino también para la división de las células 


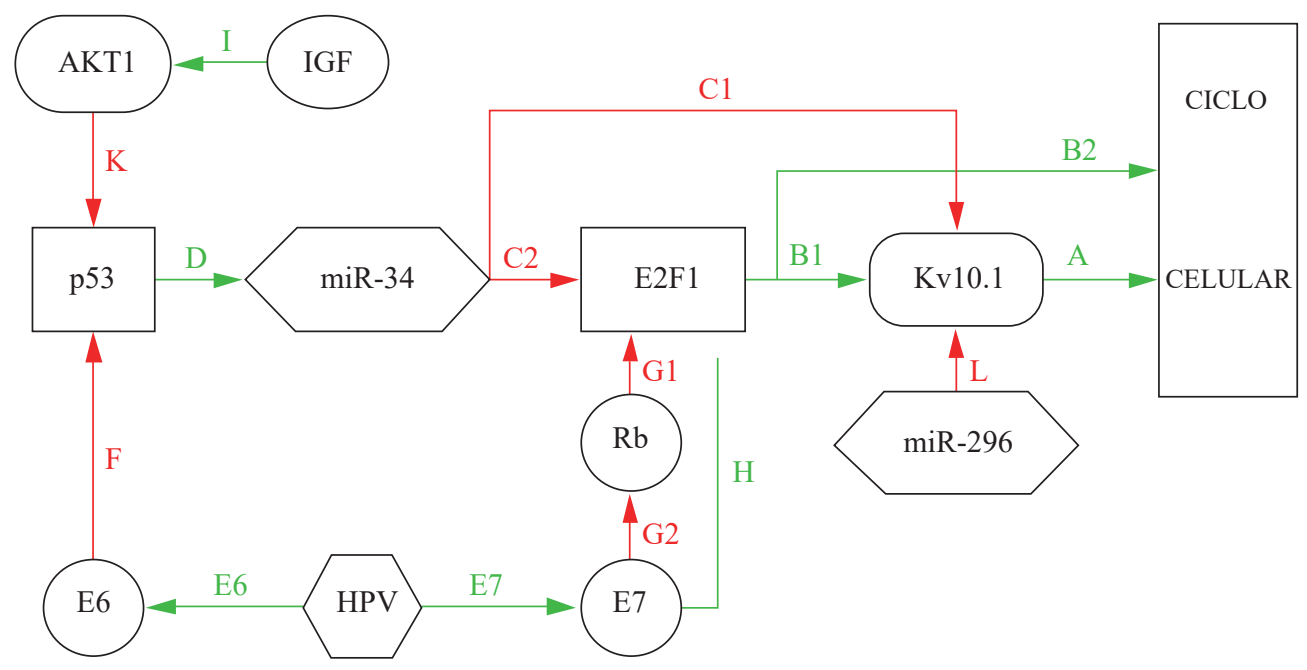

Figura 1. Esquema de las interacciones entre algunos de los componentes que regulan el ciclo celular. El esquema es muy parcial y se refiere únicamente a procesos que están involucrados en la regulación de la expresión del Kv10.1. Las flechas verdes indican aumento del producto o activación, las rojas, diminución del producto o inhibición. E2F1: factor de crecimiento; p53 y Rb: genes supresores de tumores; miR34a y miR-296: micro-ARN 34a y 296, respectivamente; HPV: virus del papiloma humano; E6 y E7: proteínas implicadas en la patogenia de HPV; IGF: factor de crecimiento similar a insulina; AKT1 Akt serina/treonina cinasa 1 o proteína cinasa B. Para más detalles, por favor referirse al texto, allí se encuentran también las referencias bibliográficas de las interacciones mencionadas.

normales (Figura 1A). El factor de crecimiento E2F1 es regulado por $p 53$ y por $R b$, dos agentes frecuentemente alterados en el cáncer. Este factor favorece tanto la expresión del Kv10.1 (B1) (Lin, et al., 2011), como la de otras proteínas esenciales para el desarrollo de la división celular (B2) (Sengupta \& Henry, 2015). Asimismo, el micro-ARN miR34 a reprime la expresión del Kv10.1 tanto directa (C1) como indirectamente mediante la reducción de la de E2F1 (C2). Una de las funciones del gen p53 es reprimir la expresión del factor de crecimiento E2F1 a través de una fuerte expresión de miR-34a (D) (Lin, et al., 2011). Una reducción o mal funcionamiento del $p 53$, evento observado en muchos tumores, causaría una reducción del miR-34a, lo cual resultaría en un aumento de E2F1 y en el consiguiente aumento del Kv10.1. Un caso concreto es la inducción de tumores por el virus del papiloma. La infección por este virus provoca la expresión de las proteínas víricas E6 (E6) y E7 (E7), las cuales afectan los oncogenes $p 53$ y $R b$, respectivamente (zur Hausen, 2002). E6 se asocia al $p 53$ e induce su degradación mediada por ubiquitinación $(\mathbf{F})$. La proteína E7 favorece la presencia del E2F1 activo (J1) al remover su inhibidor, el gen $R b(\mathbf{J 2})$. Además, E7 es capaz de unirse directamente con E2F1, activándolo independientemente de su interacción con el $R b$ (H) (Hwang, et al., 2002). Teniendo en cuenta estos resultados, no es sorprendente que Ortiz, et al. (2011) pudieran demostrar en su análisis de muestras de tejido de cérvix una frecuente expresión del Kv10.1 en tumores de cérvix y, también en tejido infectado por el virus del papiloma pero sin cáncer (Ortiz, et al., 2011).

Además del E2F1, otro factor de crecimiento está involucrado en la sobreexpresión tumoral de Kv10.1. El factor de crecimiento similar a la insulina, IGF1, es capaz de inducir una producción de Kv10.1 patológica e inducir cáncer a través de la cinasa AKT1 (I, K) (Borowiec, et al., 2007). En glioblastomas, tumores cerebrales muy malignos, el grupo de Bai, et al. (2013) logró demostrar que la sobreexpresión del Kv10.1 dependía también de una reducción del micro-ARN miR-296 (L), y que los tumores expresaban menos Kv10.1 si se les proveía de un exceso de miR-296. Asimismo, los tumores se vuelven menos resistentes a medicamentos antitumorales (Bai, et al., 2013). A partir de la figural se puede deducir que la presencia de los genes $p 53$ y $R b$ funcionales permite mantener un nivel bajo de expresión del Kv10.1. Sin embargo, este es expresado excesivamente si la presencia de $p 53$ o $R b$ es escasa o si estos no son funcionales, si hay infección con el virus del papiloma, o si el receptor del factor de crecimiento similar a la insulina está activado. De hecho, estos son eventos que en muchos casos causan cáncer. Además de los procesos aquí descritos, hay numerosos otros que debido a restricciones de espacio no se pueden detallar, por ejemplo, la regulación del Kv10.1 por el estrógeno a través de los respectivos receptores (Díaz, et al., 2009).

\section{Regulación de la división celular}

El factor de crecimiento E2F1, sus homólogos E2F2 y E2F3, así como otros procesos que no se describen aquí (por ejemplo, las ciclinas y cinasas dependientes del ciclo) forman un sistema estrechamente regulado por la división celular. El Kv10.1 es ciertamente un jugador importante en este proceso, pero no el fundamental. Se ha comprobado, sin embargo, cómo la sobreexpresión del Kv10.1 es capaz por sí sola de inducir una división celular descontrolada que favorece mucho la formación de tumores (Pardo, et al., 1999). 
Una propiedad de los tumores sólidos es su estado hipóxico, el cual induce la angiogénesis activando el factor inductor de hipoxia (Hipoxia Inducible Factor, HIF), que a su vez promueve la producción de factores proangiogénicos, como el factor de crecimiento endotelial vascular (Vascular Endotelial Growth Factor, VEGF) (Zimna \& Kurpisz, 2015). El aumento de la vascularización incrementa el suministro de oxígeno al tumor, favoreciendo así su crecimiento; es más, el aumento del oxígeno disponible es indispensable para satisfacer el elevado metabolismo de cualquier tumor que crezca rápidamente. Resulta que el Kv10.1 induce la angiogénesis aumentando el umbral de presión parcial de oxígeno en el que se inicia el incremento del HIF (Downie, et al., 2008), y por ello, confiere al tumor una ventaja metabólica, favoreciendo su crecimiento. Esto es importante en los tumores sólidos, pero no lo es tanto en los del sistema sanguíneo, como las leucemias, ya que estas no dependen de la angiogénesis para el suministro de oxígeno. Cerca de $50 \%$ de las leucemias expresan el Kv10.1. También en este caso se observa una correlación entre el nivel de expresión del canal y un pronóstico poco favorable (Agarwal, et al., 2010). Este hecho indica que el Kv10.1 debe contar con otro mecanismo, más significativo que la angiogénesis, para promover la progresión tumoral.

La división celular es una secuencia de eventos altamente coordinados y regulados por la expresión de muy diversos genes y proteínas que aseguran la duplicación fidedigna de una célula madre. Las fallas en esta regulación llevan a la ausencia de proliferación o a la formación de células con defectos genéticos. La célula madre normalmente se encuentra en la fase G0. Al iniciar la replicación entra en una fase de crecimiento (de gap), la fase G1. Sigue la fase S, llamada así por sintetizar copias de su ADN. En la siguiente fase, la G2, se producen proteínas y orgánulos. Finalmente, ocurre la fase M, en la cual las copias de ADN se separan para después terminar el ciclo con dos células en fase G1. Ya que la expresión del factor de crecimiento E2F1 es un regulador importante del ciclo celular $\mathrm{y}$, por lo tanto, su expresión varía durante el ciclo celular, la expresión del Kv10.1 también debería ser cíclica debido a su dependencia del E2F1. De hecho, al sincronizar las células al inicio de la transición entre la fases $\mathrm{G} 1$ y $\mathrm{S}$ mediante un exceso de timidina, Urrego, et al. (2016) encontraron que los niveles de expresión tanto del Kv10.1 como de las ciclinas A2 y B1 (medidas mediante electrotransferencia) exhibieron una expresión cíclica al permitir que las células reiniciaran su patrón de replicación. La expresión máxima del Kv10.1 se observó en la transición de la G2 a la M y decayó de nuevo una vez el ciclo celular se completó después de unas 24 horas. Esto aún no es prueba de una regulación directa del Kv10.1 por parte del E2F2, ya que pueden existir uno o varios pasos intermedios entre los cambios en el E2F1 y el Kv10.1. Por esta razón, Urrego, et al., insertaron una luciferasa como gen reportero bajo el control del promotor del Kv10.1, y utilizaron la cantidad de luz emitida como indicador de su expresión. Se pudo demostrar que las variaciones de luz emitida durante el ciclo celular se correlacionaban perfectamente con variaciones del Kv10.1 medidas mediante Western blot. Usando estas técnicas, e introduciendo mutaciones que evitan la interacción del E2F1 con la secuencia de la región promotora del Kv10.1, a la que normalmente se une, se lograron reducir significativamente las variaciones cíclicas del Kv10.1 a través del ciclo celular, demostrando una regulación directa del E2F1 sobre los niveles de expresión del primero. Otra evidencia independiente de esta regulación se obtiene al sobreexpresar la proteína vírica $\mathrm{E} 7$, que inhibe $R b$ (paso G2 en la figura 1) y causa, por lo tanto, un aumento del E2F1 (paso J1 en la figura 1). Como resultado, la expresión del Kv10.1 se ve significativamente aumentada. Estos hallazgos demuestran una regulación directa y periódica del Kv10.1 por E2F1 durante el ciclo celular (Urrego, et al., 2016). Una condición para la expresión cíclica de las proteínas es su degradación rápida a una escala menor del tiempo de la duración del ciclo celular, en este caso unas 24 horas. La degradación de las proteínas es iniciada, entre otras, por la ubiquitinación (Glickman \& Ciechanover, 2002). De hecho, el Kv10.1 es marcado para la degradación por ubiquitina, y se degrada en un tiempo constante de menos de 50 minutos (Kohl, et al., 2011)

Los anteriores resultados se aplican no solo a células cancerosas, sino a todas aquellas en proceso de división. Dado que existe una correlación temporal entre la expresión del Kv10.1 y, por ejemplo, la ciclina B1, se puede estudiar la expresión del primero en células no cancerosas que se dividen rápidamente, como en la cripta del colon. En la figura 2 se puede ver la expresión del Kv10.1 y de la ciclina $\mathrm{B} 1$ en las células que forman la cripta. A medida que se van diferenciando y dejan de reproducirse, tanto la ciclina B1 como el Kv10.1 disminuyen.

\section{¿Cuál es el mecanismo que permite que la sobre- expresión de Kv10.1 favorezca la división celular?}

Prácticamente todas las células tienen un cilio primario, presente en los estadios G0, G1 y, parcialmente, en las fase S y G2. Sin embargo, este cilio es reabsorbido antes de entrar en la fase $\mathrm{M}$, para volver a aparecer después de la citocinesis. $\mathrm{Al}$ igual que otros cilios, el cilio primario se ancla en un cuerpo basal, o cinetosoma, que en este caso es uno de los dos centriolos que forman el centrosoma de la célula, el cual actúa como centro de organización de los microtúbulos encargados de segregar los cromosomas durante la mitosis. Esto implica la reabsorción del cilio primario durante la fase $\mathrm{M}$, como se ha indicado anteriormente. Como consecuencia, es indispensable resorber el cilio primario para que la división celular prosiga. Curiosamente, muchas células tumorales carecen de cilio primario y, si lo exhiben, es durante un periodo muy breve (Seeley, et al., 2009). Al estudiar la localización subcelular de Kv10.1, Sánchez, et al. (2016) pudieron demostrar que el Kv10.1 se encuentra 


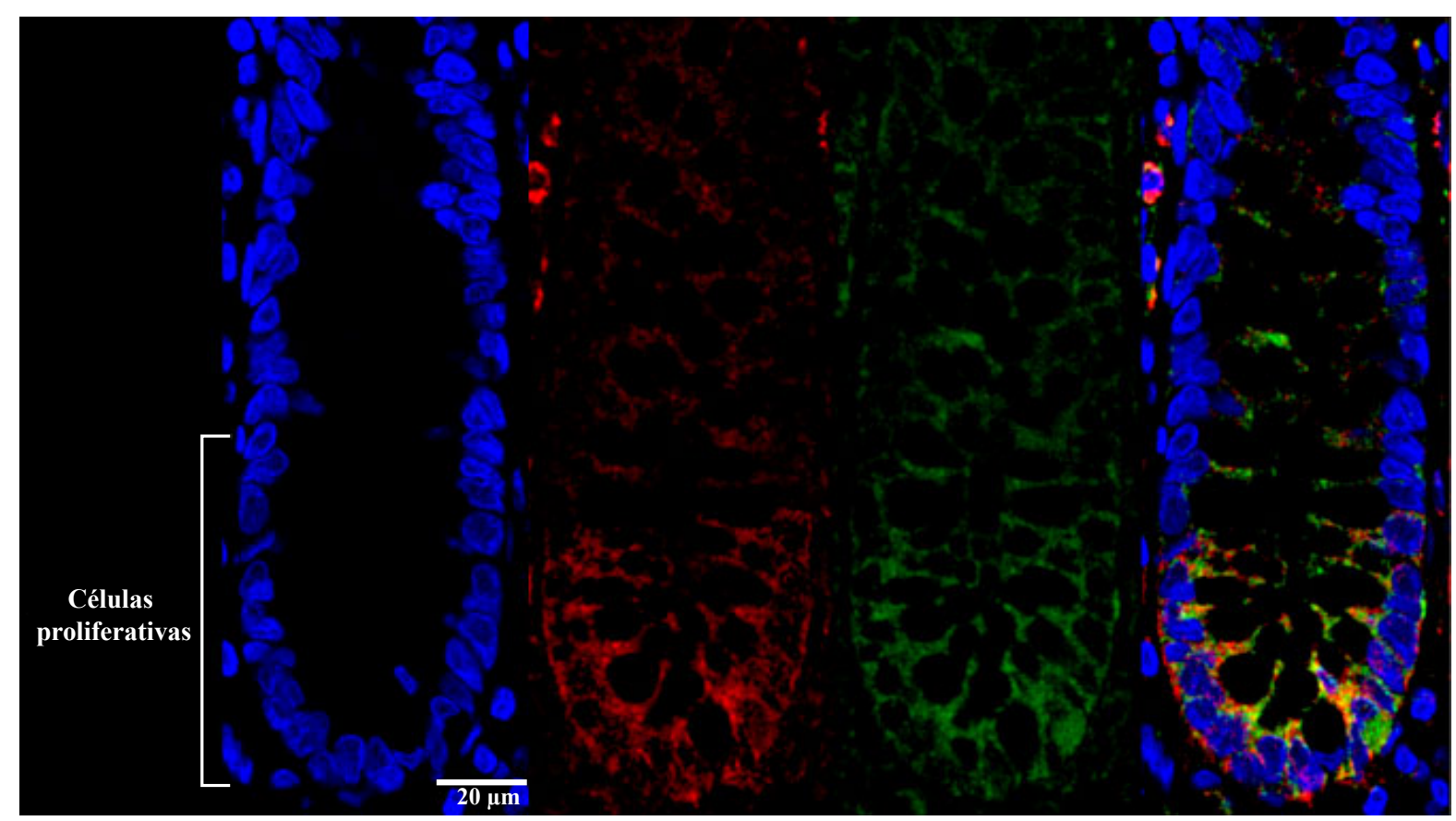

Figura 2. Imágenes de células que forman criptas en el colon. En la parte inferior se encuentran las células madre que dan lugar a una población de células que proliferan y se diferencian a medida que migran hacia la superficie de la mucosa del colon (en la parte superior de la imagen); las células maduran y dejan de replicarse en el tercio superficial de la cripta. Las células del fondo de la cripta están entre las que más rápidamente se replican en el organismo. La figura muestra una cripta teñida mediante inmunofluorescencia con marcadores de varios colores. A la izquierda, marcados con tinción TO-PRO-3, se observan los núcleos de las células en azul. A continuación, en rojo se revela la expresión de la proteína Kv10.1 con un anticuerpo específico contra ese canal (mAb62; Hemmerlein, et al., 2010) conjugado con un anticuerpo secundario marcado con AlexaFluor546. A su derecha, en verde, se observa un anticuerpo que reconoce a la ciclina B1 (rabbit anti-Cyclin B1, Cell Signaling: www. cellsignal.com/antibody/4138) conjugado con un anticuerpo secundario marcado con AlexFluor488. A la derecha se ve la superposición de las tres imágenes individuales. La combinación del color rojo con el verde resulta en el color amarillo, e indica los lugares en donde la Kv10.1 y la ciclina B1 coexisten, confirmando la presencia de Kv10.1 en las células proliferativas.

en el centrosoma, la base del cilio primario. Anteriormente se mencionó que el Kv10.1 se expresa, sobre todo, justo antes de la fase $\mathrm{M}$, antes de la resorción del cilio primario. Analizando células en proceso de división, estas se pueden diferenciar en dos grupos bien distintos: células que expresan el Kv10.1 y no tienen cilio primario, y células que tienen cilio primario, pero no expresan el Kv10.1. Pero, ¿hay una relación causal entre la expresión del Kv10.1 y la resorción del cilio primario? Sánchez, et al. lograron demostrar que la sobreexpresión del Kv10.1 inhibía la ciliogénesis, y que la reducción de su nivel de expresión mediante el uso de ARN de silenciamiento o interferencia, prolongaba la presencia del cilio primario (Sánchez, et al., 2016). Por lo tanto, el Kv10.1 promueve la endocitosis del cilio primario y explica por qué en anteriores investigaciones se demostró que el Kv10.1 interactuaba con proteínas involucradas en la endocitosis, como la cortactina (Herrmann, et al., 2012) y rabaptina 5 (Ninkovic, et al., 2012).

\section{Resumen y conclusiones}

El canal dependiente de voltaje Kv10.1, además de tener funciones como inhibidor de la excitación neuronal dependiendo de la actividad previa, tiene una función central en la regulación de la división celular y, por lo tanto, en el cáncer. Su expresión está regulada por el $p 53$ y el $R b$, dos importantes genes supresores de tumores. El Kv10.1, a su vez, regula la división celular a través de la resorción del cilio primario y se puede detectar en células que se dividen activamente, por lo cual sirve como un indicador de la presencia de tejido en crecimiento, así como de marcador precoz de la transformación maligna y de pronóstico. Sin embargo, el tejido hiperplásico también expresa el Kv10.1, en tanto que no todos los tejidos cancerosos lo sobreexpresan.

Además de su potencial diagnóstico, el Kv10.1 puede utilizarse como un blanco terapéutico, pues atacar este canal representa una vía independiente de las que actualmente se usan en los tratamientos contra el cáncer, basadas principalmente en la interferencia con los receptores de factores de crecimiento extracelulares. La combinación de estas estrategias seguramente resultaría en un tratamiento más eficaz, sobre todo porque la reducción o el bloqueo de la expresión del Kv10.1 tiene consecuencias mínimas para la fisiología normal. El astemizol, un medicamento usado como antihistamínico por millones de personas, tiene como efecto secundario disminuir las corrientes a través del Kv10.1 y ha sido eficaz en la reducción de tumores en modelos de cáncer 
in vitro y en animales (Downie, et al., 2008, Pardo, et al., 2012, García-Quiroz \& Camacho, 2011). El astemizol fue retirado del mercado debido a que aproximadamente en ocho casos por millón de pacientes y dosis producía arritmias cardiacas debido a su interacción con el Kv11.1. El Kv11.1, homólogo del Kv10.1, es uno de los tres canales responsables de la repolarización del potencial de acción del corazón. Los pacientes propensos a arritmias inducidas por astemizol se pueden detectar mediante la monitorización cardíaca, con lo cual se lo podría usar en la clínica. Para evitar las interacciones entre el Kv10.1 y el Kv11.1, sería ideal contar con agentes más selectivos del Kv10.1, por ejemplo, los anticuerpos monoclonales, los cuales se han unido con sustancias que inducen la muerte celular o apoptosis, tales como el ligando inductor de apoptosis relacionada con el factor de necrosis tumoral (TNF-Related Apoptosis-Inducing Ligand, TRAIL). Las proteínas recombinantes que resultan de la fusión entre el anticuerpo monoclonal específico contra el Kv10.1 y el TRAIL han logrado inducir apoptosis en células que expresan el Kv10.1 directamente, así como en las células a su alrededor (Hartung \& Pardo, 2016). La evidencia de la efectividad del uso del Kv10.1 como blanco contra el cáncer en tejidos in vitro y en modelos animales es completa y contundente. Los pacientes con glioblastoma multiforme o metástasis cerebrales de otros tumores primarios tienen un peor pronóstico si sus tumores expresan el Kv10.1, pero en un estudio, aquellos que fueron tratados con antidepresivos tricíclicos, también capaces de bloquear el Kv10.1, tuvieron una mayor supervivencia (Martínez, et al., 2015).

Con todos estos datos, corresponde ahora a la industria farmacéutica o a instituciones con la capacidad requerida, la realización de ensayos clínicos que transformen las pruebas preclínicas en ventajas reales para los pacientes. Probablemente debido a los altos costos que implican, no se sabe de ensayos clínicos en proceso. Los estudios descritos aquí son un ejemplo de cómo la investigación básica para entender la función de una entidad molecular desconocida ha abierto un camino prometedor para combatir el cáncer.

\section{Agradecimientos}

Este trabajo no hubiera sido posible sin la colaboración durante muchos años del profesor Luis Ángel Pardo. Agradezco también a todos los miembros del departamento de Molecular Biology of Neuronal Signals" (Göttingen, Alemania), al profesor Luis A. Pardo y a la doctora Carolina León Pinzón por sus sugerencias y la revisión del texto. Los estudios fueron posibles gracias al apoyo financiero, entre otras, de la sociedad Max Planck y de la Comunidad Europea.

\section{Conflicto de intereses}

El autor declara no tener conflictos de intereses.

\section{Referencias}

Agarwal, J.R., Griesinger, F., Stühmer, W., Pardo, L.A. 2010. The potassium channel Ether à go-go is a novel prognostic factor with functional relevance in acute myeloid leukemia. Mol Cancer. 9: 18.
Arcangeli, A., Becchetti, A. 2015. Novel perspectives in cancer therapy: Targeting ion channels. Drug Resistance Updates. 21-22: 11-19.

Bai, Y., Liao, H., Liu, T., Zeng, X., Xiao, F., Luo, L. 2013. MiR296-3p regulates cell growth and multi-drug resistance of human glioblastoma by targeting ether-a-go-go (EAG1). Eur J Cancer. 49: 710-724.

Borowiec, A.S., Hague, F., Harir, N., Guenin, S., Guerineau, F., Gouilleux, F., Roudbaraki, M., Lassoued, K., OuadidAhidouch, H. 2007. IGF-1 activates hEAG $\mathrm{K}^{+}$channels through an Akt-dependent signaling pathway in breast cancer cells: role in cell proliferation. J Cell Physiol. 212: 690-701.

Camacho, J., Sánchez, A., Stühmer, W., Pardo, L.A. 2000. Cytoskeletal interactions determine the electrophysiological properties of human EAG potassium channels. Pflugers Arch. 441: 167-174.

Díaz, L., Ceja-Ochoa, I., Restrepo-Angulo, I., Larrea, F., ÁvilaChávez, E., García-Becerra, R., Borja-Cacho, E., Barrera, D., Ahumada, E., Gariglio, P. et al. 2009. Estrogens and human papilloma virus oncogenes regulate human Ethera-go-go-1 potassium channel expression. Cancer Res. 69: 3300-3307.

Downie, B.R., Sánchez, A., Knötgen, H., Contreras-Jurado, C., Gymnopoulos, M., Weber, C., Stühmer, W., Pardo, L.A. 2008. Eag1 expression interferes with hypoxia homeostasis and induces angiogenesis in tumors. J Biol Chem. 283: 36234-36240.

García-Quiroz, J., Camacho, J. 2011. Astemizole: An old antihistamine as a new promising anti-cancer drug. Anti-Cancer Agents Med Chem. 11: 307-314.

Glickman, M.H., Ciechanover, A. 2002. The ubiquitin-proteasome proteolytic pathway: Destruction for the sake of construction. Physiol Rev. 82: 373-428.

Hartung, F., Pardo, L.A. 2016. Guiding TRAIL to cancer cells through Kv10.1 potassium channel overcomes resistance to doxorubicin. Eur Biophys J. 45: 709-719.

Hemmerlein, B., Weseloh, R.M., de Queiroz, F.M., Knötgen, H., Sánchez, A., Rubio, M.E., Martin, S., Schliephacke, T., Jenke, M., Radzun, H.J., et al. 2006. Overexpression of Eag1 potassium channels in clinical tumours. Mol Cancer. 5: 41 .

Herrmann, S., Ninkovic, M., Kohl, T., Lörinczi, E., Pardo, L.A. 2012. Cortactin controls surface expression of the voltagegated potassium channel $\mathrm{K}_{\mathrm{v}}$ 10.1. J Biol Chem. 287: 4415144163.

Hwang, S.G., Lee, D.Y., Kim, J.Y., Seo, T.G., Choe, J.H. 2002. Human papillomavirus type 16 E7 binds to E2F1 and activates E2F1-driven transcription in a retinoblastoma protein-independent manner. J Biol Chem. 277: 2923-2930.

Kamb, A., Tsengcrank, J., Tanouye, M.A. 1988. Multiple products of the Drosophila shaker gene may contribute to potassium channel diversity. Neuron. 1: 421-430.

Kohl, T., Lörinczi, E., Pardo, L.A., Stühmer, W. 2011. Rapid internalization of the oncogenic K+ channel Kv10.1. PLoS ONE. 6: e26329.

Lastraioli, E., Perrone, G., Sette, A., Fiore, A., Crociani, O., Manoli, S., D'Amico, M., Masselli, M., Iorio, J., Callea, M., et al. 2015. hERG1 channels drive tumour malignancy and may serve as prognostic factor in pancreatic ductal adenocarcinoma. Brit J Cancer. 112: 1076-1087. 
Lin, H.X., Li, Z., Chen, C., Luo, X.B., Xiao, J.N., Dong, D.L., Lu, Y.J., Yang, B.F., Wang, Z.G. 2011. Transcriptional and post-transcriptional mechanisms for oncogenic overexpression of ether a go-go $\mathrm{K}+$ channel. PLoS ONE. 6: 10.

Martínez, R., Stühmer, W., Martin, S., Schell, J., Reichmann, A., Rohde, V., Pardo, L. 2015. Analysis of the expression of Kv10.1 potassium channel in patients with brain metastases and glioblastoma multiforme: Impact on survival. BMC Cancer. 15: 1-9.

Menéndez, S.T., Villaronga, M.A., Rodrigo, J.P., ÁlvarezTeijeiro, S., García-Carracedo, D., Urdinguio, R.G., Fraga, M.F., Pardo, L.A., Gutiérrez Viloria, C., Suárez, C., García-Pedrero, J.M. 2012. Frequent aberrant expression of the human ether à go-go (hEAG1) potassium channel in head and neck cancer: Pathobiological mechanisms and clinical implications. J Mol Med (Berl). 90: 1173-1184.

Mortensen, L.S., Schmidt, H., Farsi, Z., Barrantes-Freer, A., Rubio, M.E., Ufartes, R., Eilers, J., Sakaba, T., Stuehmer, W., Pardo, L.A. 2015. Kv10.1 opposes activity-dependent increase in $\mathrm{Ca}^{2+}$ influx into the presynaptic terminal of the parallel fibre-Purkinje cell synapse. J Physiol. 593: 181-196.

Ninkovic, M., Mitkovski, M., Kohl, T., Stühmer, W., Pardo, L.A. 2012. Physical and functional interaction of $\mathrm{K}_{\mathrm{v}} 10.1$ with Rabaptin-5 impacts ion channel trafficking. FEBS Lett. 586: 3077-3084.

Ouadid-Ahidouch, H., Ahidouch, A., Pardo, L.A. 2016. Kv10.1 $\mathrm{K}+$ channel: From physiology to cancer. Pflugers Arch. 468: 751-762.

Ortiz, C.S., Montante-Montes, D., Saqui-Salces, M., Hinojosa, L.M., Gamboa-Domínguez, A., Hernández-Gallegos, E., Martínez-Benítez, B., Solís-Pancoatl, M.D.R., GarcíaVilla, E., Ramírez, A., et al. 2011. Eag1 potassium channels as markers of cervical dysplasia. Oncol Rep. 26: 1377-1383.

Pardo, L.A., del Camino, D., Sánchez, A., Alves, F., Brüggemann, A., Beckh, S., Stühmer, W. 1999. Oncogenic potential of EAG K ${ }^{+}$channels. EMBO J. 18: 5540-5547.

Pardo, L.A., Gómez-Varela, D., Major, F., Sansuk, K., Leurs, R., Downie, B.R., Tietze, L.F., Stühmer, W. 2012. Approaches targeting K(V)10.1 open a novel window for cancer diagnosis and therapy. Curr Med Chem. 19: 675-682.

Pardo, L.A., Stühmer, W. 2014. The roles of K+ channels in cancer. Nat Rev Cancer. 14: 39-48.

Pongs, O., Kecskemethy, N., Muller, R., Krahjentgens, I., Baumann, A., Kiltz, H.H., Canal, I., Llamazares, S., Ferrus, A. 1988. Shaker encodes a family of putative potassium channel proteins in the nervous system of Drosophila. EMBO J. 7: 1087-1096.

Sánchez, A., Urrego, D., Pardo, L.A. 2016. Cyclic expression of the voltage-gated potassium channel KV10.1 promotes disassembly of the primary cilium. EMBO Rep. 17: 708-723.
Schwarz, T.L., Tempel, B.L., Papazian, D.M., Jan, Y.N., Jan, L.Y. 1988. Multiple potassiium-channel components are produced by alternative splicing at the Shaker locus in Drosophila. Nature. 331: 137-142.

Seeley, E.S., Carriere, C., Goetze, T., Longnecker, D.S., Korc, M. 2009. Pancreatic cancer and precursor pancreatic intraepithelial neoplasia lesions are devoid of primary cilia. Cancer Res. 69: 422-430.

Sengupta, S., Henry, R.W. 2015. Regulation of the retinoblastomaE2F pathway by the ubiquitin-proteasome system. Biochim Biophys Acta-Gene Regul Mech. 1849: 1289-1297.

Stansfeld, C.E., Röper, J., Ludwig, J., Weseloh, R.M., Marsh, S.J., Brown, D.A., Pongs, O. 1996. Elevation of intracellular calcium by muscarinic receptor activation induces a block of voltage-activated rat Ether-à-go-go channels in a stably transfected cell line. Proceedings of the National Academy of Sciences. 93: 9910-9914.

Terlau, H., Ludwig, J., Steffan, R., Pongs, O., Stühmer, W., Heinemann, S. 1996. Extracellular Mg2+ regulates activation of rat eag potassium channel. Pflügers Archiv European Journal of Physiology. 432: 301-312.

Stühmer, W., Stocker, M., Sakmann, B., Seeburg, P., Baumann, A., Grupe, A., Pongs, O. 1988. Potassium channels expressed from rat-brain CDNA have delayed retifier properties. FEBS Lett. 242: 199-206.

Ufartes, R., Schneider, T., Mortensen, L.S., de Juan Romero, C., Hentrich, K., Knoetgen, H., Beilinson, V., Moebius, W., Tarabykin, V., Alves, F., et al. 2013. Behavioural and functional characterization of Kv10.1 (Eag1) knockout mice. Hum Mol Genet. 22: 2247-2262.

Urrego, D., Movsisyan, N., Ufartes, R., Pardo, L.A. 2016. Periodic expression of Kv10.1 driven by pRb/E2F1 contributes to $\mathrm{G} 2 / \mathrm{M}$ progression of cancer and non-transformed cells. Cell Cycle. 15: 799-811.

Wadhwa, S., Wadhwa, P., Dinda, A.K., Gupta, N.P. 2009. Differential expression of potassium ion channels in human renal cell carcinoma. Int Urol Nephrol. 41: 251-257.

Warmke, J.W., Ganetzki, B. 1994. A family of potassium channel genes related to eag in Drosophila and mammals. Proc Natl Acad Sci USA. 91: 3438-3442.

Zimna, A., Kurpisz, M. 2015. Hypoxia-inducible factor-1 in physiological and pathophysiological angiogenesis: Applications and therapies. Biomed Res Int. 2015: 549412.

zur Hausen, H. 2002. Papillomaviruses and cancer: From basic studies to clinical application. Nature Rev Cancer. 2: 342350 . 